\title{
Energy Analysis of Bare Electrodynamic Tethers
}

\author{
M. Sanjurjo-Rivo* and J. Peláez ${ }^{\dagger}$ \\ E. T. S. I. Aeronáuticos, 28040 Madrid, Spain
}

\begin{abstract}
The design of an electrodynamic tether is a complex task that involves the control of dynamic instabilities, optimization of the generated power (or the descent time in deorbiting missions), and minimization of the tether mass. The electrodynamic forces on an electrodynamic tether are responsible for variations in the mechanical energy of the tethered system and can also drive the system to dynamic instability. Energy sources and sinks in this system include the following: 1) ionospheric impedance, 2) the potential drop at the cathodic contactor, 3 ) ohmic losses in the tether, 4) the corotational plasma electric field, and 5) generated power and/or 6) input power. The analysis of each of these energy components, or bricks, establishes parameters that are useful tools for tether design. In this study, the nondimensional parameters that govern the orbital energy variation, dynamic instability, and power generation were characterized, and their mutual interdependence was established. A space-debris mitigation mission was taken as an example of this approach for the assessment of tether performance. Numerical simulations using a dumbbell model for tether dynamics, the International Geomagnetic Reference Field for the geomagnetic field, and the International Reference Ionosphere for the ionosphere were performed to test the analytical approach. The results obtained herein stress the close relationships that exist among the velocity of descent, dynamic stability, and generated power. An optimal tether design requires a detailed tradeoff among these performances in a real-world scenario.
\end{abstract}

\section{Nomenclature}

$A_{t} \quad=$ area of the tether cross section

$a=\quad$ semimajor axis of the tethered system orbit

$E \quad=$ mechanical energy of the tethered system

$E_{m} \quad=$ motional electric field

$e=$ eccentricity of the tethered system orbit

$\boldsymbol{F}_{\text {grav }} \quad=$ resultant of the gravitational force

$\boldsymbol{F}_{p} \quad=$ resultant of the perturbation forces

$\hat{f}^{p} \quad=$ nondimensional Lorentz torque

$\circlearrowleft(\gamma)=$ switching function between passive and active tethers

$\boldsymbol{H}=$ angular momentum

$h \quad=$ thickness of a thin-tape tether

$I=$ current along the tether

$\bar{I}_{G} \quad=$ central inertia tensor

$I_{\mathrm{sc}} \quad=$ short-circuit current

$i\{$ or $b\}=$ inclination of the tethered system orbit

$i=$ nondimensional current along the tether

$L=$ tether length

$L_{*} \quad=$ characteristic tether length

$\ell_{t} \quad=$ nondimensional tether length

$\boldsymbol{M}_{\text {grav }}=$ gravitational torque on the center of mass

$\boldsymbol{M}_{p}=$ torque of the perturbation forces

$m \quad=$ mass of the tethered system

$m_{e} \quad=$ electron mass

$m_{i} \quad=$ ion mass

$m_{1} \quad=$ point mass at the lower end of the tether

$m_{2} \quad=$ point mass at the upper end of the tether

$n_{\infty}=$ density of ionospheric plasma

$p_{t}=$ tether perimeter

$q \quad=$ electron charge

$R \quad=$ tether radius

$R_{T} \quad=$ tether resistance

Received 20 November 2009; revision received 3 June 2010; accepted for publication 10 September 2010. Copyright (C) 2010 by Manuel Sanjurjo-Rivo. Published by the American Institute of Aeronautics and Astronautics, Inc., with permission. Copies of this paper may be made for personal or internal use, on condition that the copier pay the $\$ 10.00$ per-copy fee to the Copyright Clearance Center, Inc., 222 Rosewood Drive, Danvers, MA 01923; include the code 0748-4658/11 and $\$ 10.00$ in correspondence with the CCC.

*Ph.D., Pza. Cardenal Cisneros 3; m. sanjurjo@upm.es.

${ }^{\dagger}$ Associate Professor, Pza. Cardenal Cisneros 3; j.pelaez@upm.es. Member AIAA. $r$
$T_{d}$
$u$
$V$
$V_{\mathrm{cc}}$
$V_{\mathrm{grav}}$
$V_{\mathrm{pl}}$
$V_{t}$
$\tilde{V}_{\mathrm{cc}}$
$v^{d s}$
$v_{G}$
$v_{p l}$
$W_{f e}^{d s}$
$Z_{T}$
$\alpha$
$\gamma$
$\epsilon$
$\tilde{\epsilon}$
$\eta_{t}$
$\theta$
$\mu$
$\nu$
$\rho$
$\sigma$
$\Phi$
$\phi$
$\varphi$
$\Omega$
$\Omega_{\mathrm{an}}$
$\omega$

$=$ position vector

$=$ time of descent

$=$ tether line unit vector

$=$ bias voltage of tether with respect to plasma

$=$ potential drop at cathodic contactor

= gravitational potential energy

= plasma voltage

$=$ tether voltage

$=$ nondimensional potential drop at the cathodic contactor

$=$ velocity of a tether element

$=$ inertial velocity of the tethered system

$=$ plasma velocity

$=$ power dissipated by electrodynamic forces in a tether element

$=$ cathodic electric load

$=$ arbitrary value

$=$ mass angle

$=$ input voltage applied to tether

$=$ nondimensional input voltage

$=$ deorbiting efficiency

$=$ in-plane libration angle

$=$ ion mass ratio

$=$ true anomaly of the tethered system orbit

$=$ density

$=$ tether conductivity

$=$ local bias potential between tether and plasma

$=$ out-of-plane libration angle

$=$ nondimensional potential bias along the tether

$=$ nondimensional electric load

$=$ right ascension of the node of the tethered system orbit

$=$ angular velocity of the tether relative to the inertial frame

$=$ argument of the periapsis of the tethered system orbit

Subscripts

$A=$ anodic end of the tether

$B=$ zero-potential point in the tether

$C=$ cathodic end of the tether

$\mathrm{CC}=$ cathodic contactor

$\mathrm{CF}=$ corotational electric field 


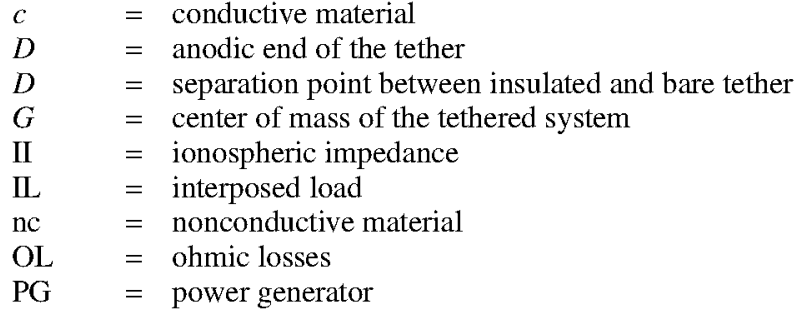

\section{Introduction}

$\mathbf{T}$ HE development of the electrodynamic tether (EDT) concept dates back to the $1960 \mathrm{~s}$, wherein the original motivation was the use of Alfven waves to provide thrust. This idea appeared for the first time in [1]. Since this original work the two-fold character of EDTs has been revealed, that is, as scientific instruments to investigate the ionosphere as well as devices that are capable of generating power or providing propulsion. This last aspect of EDTs was stimulated by Alfven [2]. A turning point in the consideration of EDTs was a paper by Martinez-Sanchez and Hastings [3], which provides for the first time a detailed assessment of the feasibility of these devices from a multidisciplinary perspective. Eventually, this technology was successfully tested in the TSS-1R mission. From then on, the appeal of these devices has centered on power generation or thrust production with little propellant consumption.

The research that has been carried out to date in this field is significant. Recent books and reviews [4,5] have summarized the current activity in this field. In addition to these reviews, two recent papers have clarified the possibilities of and the concerns with EDT technology. The first paper [6] is a compact but complete guide to EDTs, especially concerning those aspects that relate to current collection. This paper also outlines several interesting possible applications and gives insight into their primary features. The second paper [7] analyzes the feasibility of EDTs in space stressing the drawbacks or challenges they may face. According to the author, the primary technological problems that EDTs must solve are the following: long-term dynamic stability, survivability, plasma contact and deployment analysis. The current research activity in this area is focused on solving these issues.

These reports highlight the amount, quality, and multidisciplinary character of the studies in this field. Research dealing with EDT operation covers a broad spectrum that ranges from plasma physics to dynamic systems and control. In addition, EDTs can work in two different regimes: active, when the EDT has a power source, and passive, when there is no power source. The former generally produces an orbital rise of the system, whereas the latter generally drives an orbital descent. Because of the different behaviors of EDT in the active and passive regimes, analyses are usually focused on only one of these regimes.

In this study, tether operation is considered from a different perspective: the energy analysis point of view. The EDT is regarded as a device for exchanging energy between mechanical and electrical forms. The aim of this paper is to provide a global framework to study EDT operation. The energy perspective is global because both passive and active tethers can be analyzed with the same methodology and this analysis links several important aspects of EDT performance. The approach presented here connects the factors that participate in current collection, dynamic instability and orbital transfer. Likewise, energy analysis of the tether provides information about the power that can be recovered in a descent trajectory and the power needed to perform any other orbital transfer. To simplify the dynamic analysis, we use the dumbbell model throughout this work.

Following this introduction, current collection in a bare EDT is reviewed; this is necessary to understand several features of the subsequent analysis. A detailed analysis of the power that is capable of being developed by electrodynamic forces is then presented. In the second part of the paper, we analyze a particular case of EDT operation (a debris-mitigation mission) to demonstrate the capabilities of our tools. Such a mission was selected for several reasons. First, EDTs are a highly effective technology for use as deorbiting devices
[8-12]. Moreover, their ability to operate in different modes allows them to be used not only for orbital decay but also as orbit-transfer [13] or power generation systems [3]. Finally, the conclusions of this work are summarized.

\section{Current Collection in Bare EDTs}

A short review of the fundamentals of current collection in bare EDTs is required to understand the following discussion. This section describes the computation of the current that flows along the cable, following a previous work that only covers passive tethers [14].

Bare EDTs have been proposed as an alternative to conventional plasma contactors in [15]. They represent a simple and robust solution to current collection in highly rarefied plasmas. Subsequent papers have explored their operational regime [orbit motion limit (OML)] [10], validity range [16], and other relevant issues [17,18].

In this study, it was assumed that the tether works within the OML regime, in short, its perimeter size is sufficiently small. The boundary conditions at the tether ends influence the collected current. Therefore, the electrical devices that are attached to the tether play a role in the boundary conditions. For passive tethers, an interposed load will be placed just before the cathodic contactor. For active tethers, in addition to the cathodic contactor, it is assumed that a power generator is connected at the end of the cathodic segment. The possibility of including an insulated segment in the cable will be contemplated for the latter case. In this situation, there are two distinct segments (anodic and cathodic) and the equations that govern the current collection process in both segments and for both kinds of tethers are as follows:

1) Passive tether:

a) Segment $A B$ (anodic), $\Phi>0$ :

$$
\begin{gathered}
\frac{\mathrm{d} I}{\mathrm{~d} s}=2 R q n_{\infty} \sqrt{\frac{2 e}{m_{e}} \Phi} \quad \frac{\mathrm{d} \Phi}{\mathrm{d} s}=\frac{I}{\sigma A_{t}}-E_{m} \quad s=0 \\
I=0 \quad s=s_{B} ; \quad \Phi=0
\end{gathered}
$$

b) Segment $B C$ (cathodic), $\Phi<0$ :

$$
\begin{gathered}
\frac{\mathrm{d} I}{\mathrm{~d} s}=-2 R q n_{\infty} \mu \sqrt{\frac{2 e}{m_{e}}|\Phi|} \\
\frac{\mathrm{d} \Phi}{\mathrm{d} s}=\frac{I}{\sigma A_{t}}-E_{m} \\
s=s_{B} ; \quad \Phi=0
\end{gathered}
$$

c) Boundary conditions:

$$
V_{\mathrm{cc}}+Z_{T} I_{C}=E_{m}\left(L-s_{B}\right)-\int_{s_{B}}^{L} \frac{I(x)}{\sigma A_{t}} \mathrm{~d} x
$$

For passive tethers, $s$ is the measured distance from the anodic end, and it ranges over the interval $\left[0, s_{B}\right]$ in the anodic segment, $A B$, with $s \in\left[s_{B}, L\right]$ in the cathodic segment $B C$ (see Fig. 1).

2) Active tether:

a) Segment $D A$ (cathodic), $\Phi<0$ :

$$
\begin{gathered}
\frac{\mathrm{d} I}{\mathrm{~d} s}=-2 R q n_{\infty} \mu \sqrt{\frac{2 e}{m_{e}}|\Phi|} \\
\frac{\mathrm{d} \Phi}{\mathrm{d} s}=\frac{I}{\sigma A_{t}}+E_{m}
\end{gathered}
$$




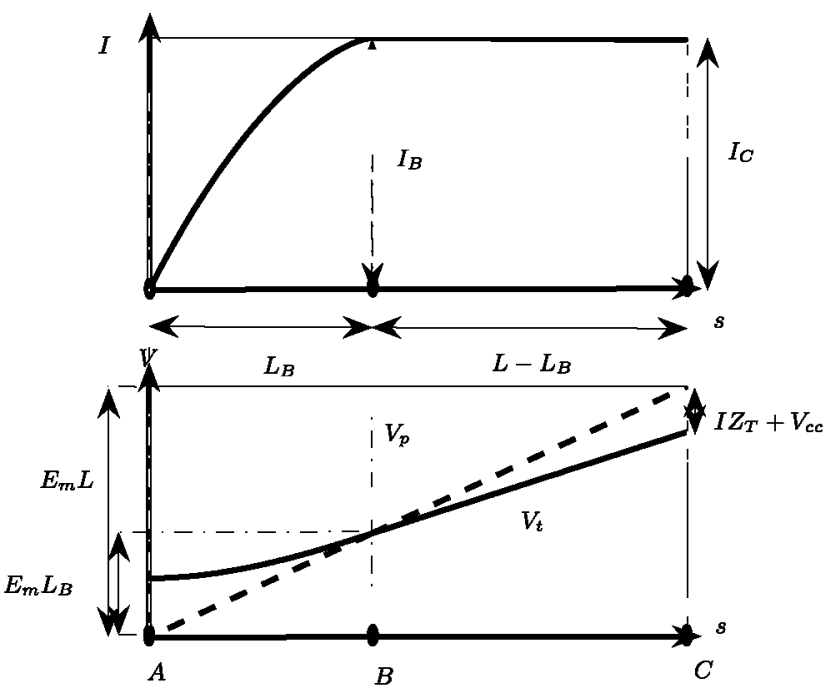

Fig. 1 The current and potential profiles in a passive tether.

$$
\begin{array}{cc}
s=0 ; & I=0 \\
s=s_{D} ; & \Phi=0
\end{array}
$$

b) Segment $B D$ (anodic), $\Phi>0$ :

$$
\begin{array}{ll}
\frac{\mathrm{d} I}{\mathrm{~d} s}=2 R q n_{\infty} \sqrt{\frac{2 e}{m_{e}} \Phi} \quad \frac{\mathrm{d} \Phi}{\mathrm{d} h}=\frac{I}{\sigma A_{t}}+E_{m} \\
s=s_{D} ; \quad \Phi=0 \quad s=s_{B} ; \quad I=I_{C} ; \quad \Phi=\Phi_{C}
\end{array}
$$

c) Boundary conditions:

$$
\epsilon-V_{\mathrm{cc}}=E_{m}\left(L-s_{D}\right)+I_{C} \frac{L-s_{B}}{\sigma A_{t}}+\int_{s_{D}}^{s_{B}} \frac{I(x)}{\sigma A_{t}} \mathrm{~d} x
$$

For active tethers, $s$ is the measured distance from the cathodic end. For the anodic segment, $B D, s$ ranges over the interval $\left[s_{B}, s_{D}\right]$, with $s \in\left[0, s_{B}\right]$ for the cathodic segment (see Fig. 2).

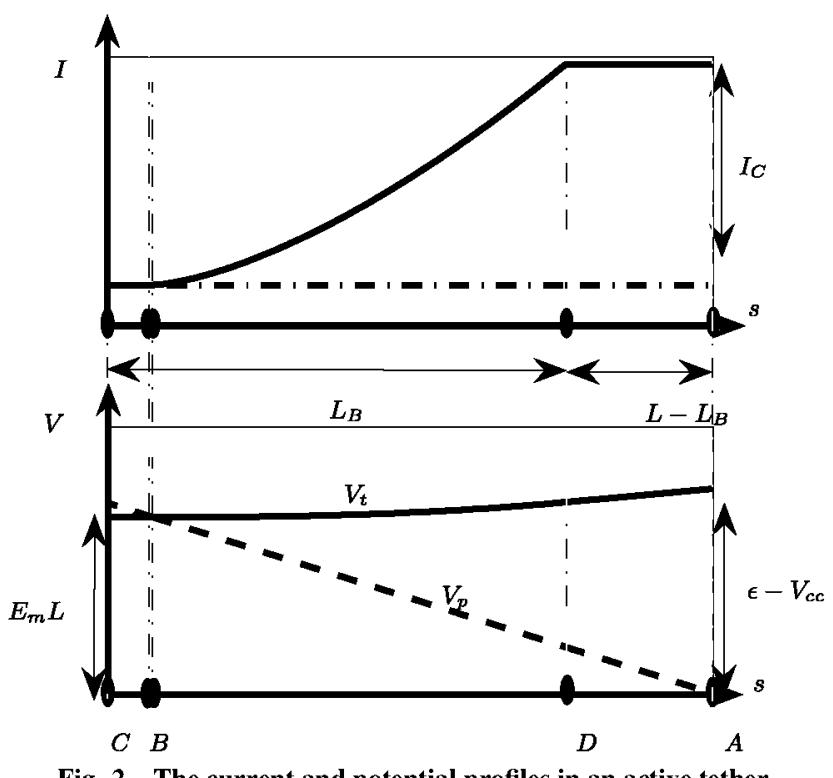

Fig. 2 The current and potential profiles in an active tether.
The tether-current profile $I(s)$ and the potential drop between the tether and plasma $\Phi(s)=V_{t}-V_{p}$ are the dependent variables. The parameters that are involved in the differential equations are the electrical conductivity, $\sigma$; electron charge, $q$; perimeter of the tether cross-section, $p_{t}$; cross-section of the conductive portion of the tether, $A_{t}$; electron mass, $m_{e}$; ion mass, $m_{i} ; \mu=\sqrt{m_{e} / m_{i}}$; induced electric field, $E_{m}$ [see Eq. (32) next]; and ionospheric plasma density, $n_{\infty}$. Note that external fields provide the free parameters of $E_{m}$ and $n_{\infty}$, which ultimately determine the electron-collection process. Additional parameters appear in the boundary conditions: these are the potential bias at the cathodic contactor, $V_{\mathrm{cc}}$, the interposed load, $Z_{T}$, and the potential rise of the power generator, $\epsilon$. Because the length of the cathodic segment is unknown (or, conversely, the anodic segment), the circuit equation (boundary conditions) closes the problem.

Figures 1 and 2 depict representations of the current and bias profiles for passive and active tethers, respectively. The significant points along the tethers are marked and named in these figures.

The use of nondimensional variables will help to elucidate the problem in a more straightforward form. The nondimensional description most frequently used (which is closely followed here) was derived in a seminal paper by Sanmartín et al. [15].

First, the characteristic length, $L_{*}$, which is given by

$$
L_{*}=\frac{\left(m_{e} E_{m}\right)^{1 / 3}}{q 2^{7 / 3}}\left(3 \pi \frac{\sigma h_{t}}{n_{\infty}}\right)^{2 / 3}
$$

is introduced. This characteristic length depends on: 1) the tether parameters $\left.\left(\sigma, h_{t}\right), 2\right)$ physical constants $\left(e, m_{e}\right)$ and, 3) environmental conditions through $E_{m}, n_{\infty}$. The magnitude $h_{t}$ is the ratio $h_{t}=2 A_{t} / p_{t}$. If the tether is a wire, $h_{t}$ is equal to the tether radius, whereas if the tether is a tape, $h_{t} \approx h$ is its thickness. The value of $L_{*}$ gauges the ohmic effects on the EDT [15], larger ohmic effects lead to shorter characteristic lengths. Scaling with this length, we obtain

$$
\xi=\frac{s}{L_{*}} \in\left[0, \ell_{t}\right], \quad \text { being } \ell_{t}=\frac{L}{L_{*}}
$$

The characteristic current is the short-circuit current, that is, $I_{\mathrm{sc}}=\sigma E_{m} A_{t}$. A characteristic voltage drop that is equal to the bias that is induced by the electric field along the characteristic length, $E_{m} L_{*}$, is used. Thus, the nondimensional forms of the dependent variables $i$ and $\varphi$ are as follows:

$$
i(\xi)=I / I_{\mathrm{sc}} \quad \varphi=\Phi /\left(E_{m} L_{*}\right)
$$

The parameters that relate to electrical devices take the following nondimensional forms:

$$
\begin{gathered}
\Omega=\frac{Z_{T}}{R_{T}}, \quad \text { being } R_{T} \equiv \frac{L}{\sigma A_{t}} \\
\tilde{\epsilon}=\frac{\epsilon}{E_{m} L}, \quad \tilde{V}_{\mathrm{cc}}=\frac{V_{\mathrm{cc}}}{E_{m} L}
\end{gathered}
$$

It is important to note that $R_{T}$ is the electric resistance of the tether. Regarding the nomenclature, $\left(\varphi_{A}, i_{A}\right)$ stands for the values of $(\varphi, i)$ at point $A$ of the tether and so on.

\section{Analysis}

\section{A. Mechanical Energy}

To clarify the operation of the tether as an energy-exchange device, some previous considerations about the energy of the orbital system are required. Let $E$ be the total mechanical energy of the system (kinetic energy plus potential energy). The kinetic energy can be split into the energy of the center of mass (orbital motion) and the rotational energy (attitude dynamics):

$$
E=\frac{1}{2} m v_{G}^{2}+\frac{1}{2} \omega \cdot \overline{\bar{I}}_{G} \cdot \omega+V_{\text {grav }}
$$


Here, $v_{G}$ is the inertial velocity of the center of mass, $G, \omega$ is the angular velocity of the tether relative to the inertial frame, $\overline{\bar{I}}_{G}$ is the central inertia tensor, and $V_{\text {grav }}$ is the gravitational potential energy. Here, the objective is to establish the mechanisms that determine the evolution of these terms. Consequently, the energy-evolution equation is derived next.

Let $\boldsymbol{F}_{\text {grav }}$ be the resultant of the gravitational forces that act on the system. From the linear momentum equation and taking the dot product with $v_{G}$, the evolution of the mechanical energy of the center of mass can be obtained:

$$
m \frac{\mathrm{d} \boldsymbol{v}_{G}}{\mathrm{~d} t}=\boldsymbol{F}_{\mathrm{grav}}+\boldsymbol{F}_{p} \Rightarrow \frac{\mathrm{d}}{\mathrm{d} t}\left(\frac{1}{2} m v_{G}^{2}\right)=\boldsymbol{F}_{\mathrm{grav}} \cdot \boldsymbol{v}_{G}+\boldsymbol{F}_{p} \cdot \boldsymbol{v}_{G}
$$

Here, $\boldsymbol{F}_{p}$ is the resultant all of the perturbation forces.

Let $\boldsymbol{M}_{\text {grav }}$ be the gravitational torque on the center of mass $G$. From the angular momentum equation and taking the dot product with $\omega$, the evolution of the rotational energy can be obtained:

$$
\begin{aligned}
& \frac{\mathrm{d}}{\mathrm{d} t}\left(\boldsymbol{H}_{G}\right)=\boldsymbol{M}_{\text {grav }}+\boldsymbol{M}_{p} \Rightarrow \frac{\mathrm{d}}{\mathrm{d} t}\left(\frac{1}{2} \boldsymbol{\omega} \cdot \overline{\bar{I}}_{G} \cdot \boldsymbol{\omega}\right) \\
& \quad=\boldsymbol{M}_{\text {grav }} \cdot \boldsymbol{\omega}+\boldsymbol{M}_{p} \cdot \boldsymbol{\omega}
\end{aligned}
$$

Here, $\boldsymbol{M}_{p}$ is the torque at the center of mass $G$ due to the perturbation forces that act on the system. In the next section, each term on the right-hand side of Eqs. (17) and (18) is discussed in detail.

By adding relations (17) and (18) the equation for the total energy of the system is obtained:

$$
\frac{\mathrm{d} E}{\mathrm{~d} t}=\boldsymbol{F}_{p} \cdot \boldsymbol{v}_{G}+\boldsymbol{M}_{p} \cdot \boldsymbol{\omega}
$$

where the relation

$$
\frac{\mathrm{d} V_{\text {grav }}}{\mathrm{d} t}=-\boldsymbol{F}_{\text {grav }} \cdot \boldsymbol{v}_{G}-\boldsymbol{M}_{\text {grav }} \cdot \boldsymbol{\omega}
$$

has been taken into account. Note that if the main perturbation is due to electrodynamic forces, the right-hand side of Eq. (19) can be positive or negative depending on the tether operation regime.

\section{B. Power of the Electrodynamic Forces}

Let $\boldsymbol{B}$ be the magnetic field at the center of mass $G$ of the system (we neglect the variation of $\boldsymbol{B}$ along the tether). The power that is generated by the electrodynamic forces in a tether element is

$$
\dot{W}_{f e}^{d s}=\boldsymbol{v}^{d s} \cdot(\boldsymbol{u} \times \boldsymbol{B}) I_{e}(s) d s
$$

Here $s$ is the measured distance along the tether from the center of mass $G$. In the dumbbell model, the velocity field is expressed as

$$
v^{d s}=v_{G}+\omega \times r
$$

where $r$ is the position vector of the mass element in the orbital frame. Let $\boldsymbol{u}$ be a unit vector along the tether. Taking into account the relations

$$
\begin{gathered}
\omega=\boldsymbol{u} \times \dot{\boldsymbol{u}}+\alpha \boldsymbol{u} \\
\boldsymbol{r}=s \boldsymbol{u}, \quad s \in[-L(1-\circlearrowleft(\gamma)), L \circlearrowleft(\gamma)]
\end{gathered}
$$

the velocity of the tether element is

$$
\boldsymbol{v}^{d s}=\boldsymbol{v}_{G}+s \dot{\boldsymbol{u}}
$$

Remarks: In the expression for angular velocity, $\alpha$ is an arbitrary value. In addition, $\Theta(\gamma)$ is equal to $\cos ^{2} \gamma$ for passive tethers and $\sin ^{2} \gamma$ for active tethers. In turn, the definition of $\gamma$ is as follows: $m \cos ^{2} \gamma=m_{1}+m_{T} / 2$ and $m \sin ^{2} \gamma=m_{2}+m_{T} / 2\left(m_{1}\right.$ is the point mass at the lower end and $m_{2}$ is the point mass at the upper end of the tether). The direction of the unit vector $\boldsymbol{u}$ always corresponds to the direction of the tether from the anodic end to the cathodic end. Consequently, the direction of the vector $\boldsymbol{u}$ of an active tether is the opposite to the direction of the same tether working in the passive regime.

The power developed by the elemental electrodynamic force becomes

$$
\dot{W}_{f e}^{d s}=v_{G} \cdot(\boldsymbol{u} \times \boldsymbol{B}) I_{e}(s) d s+\dot{\boldsymbol{u}} \cdot(\boldsymbol{u} \times \boldsymbol{B}) s I_{e}(s) d s
$$

Thus, the overall power on the cable will be

$$
\begin{gathered}
\dot{W}_{f e}=\boldsymbol{v}_{G} \cdot(\boldsymbol{u} \times \boldsymbol{B}) \int_{-L(1-G(\gamma))}^{L G(\gamma)} I_{e}(x) \mathrm{d} x \\
+\dot{\boldsymbol{u}} \cdot(\boldsymbol{u} \times \boldsymbol{B}) \int_{-L(1-\circlearrowleft(\gamma))}^{L G(\gamma)} x I_{e}(x) \mathrm{d} x
\end{gathered}
$$

The electrodynamic force, $\boldsymbol{F}_{p}$, and the torque, $\boldsymbol{M}_{p}$, that act on the cable are given by

$$
\begin{gathered}
\boldsymbol{F}_{p}=(\boldsymbol{u} \times \boldsymbol{B}) \int_{-L(1-\mathfrak{G}(\gamma))}^{L \mathfrak{G}(\gamma)} I_{e}(x) \mathrm{d} x \\
\boldsymbol{M}_{p}=(\boldsymbol{u} \times[\boldsymbol{u} \times \boldsymbol{B}]) \int_{-L(1-\mathfrak{G}(\gamma))}^{L \mathbb{G}(\gamma)} x I_{e}(x) \mathrm{d} x
\end{gathered}
$$

The power can be expressed as follows:

$$
\dot{W}_{f e}=\boldsymbol{F}_{p} \cdot \boldsymbol{v}_{G}+\boldsymbol{M}_{p} \cdot \boldsymbol{\omega}
$$

Equations (17) and (18) permit us to evaluate the distribution of the power that is created by the electrodynamic forces between the orbital motion and the attitude dynamics, however, for a further insight, it is convenient to express the term $\boldsymbol{v}_{G} \cdot(\boldsymbol{u} \times \boldsymbol{B})$ in a different manner:

$$
\begin{aligned}
& \boldsymbol{v}_{G} \cdot(\boldsymbol{u} \times \boldsymbol{B})=\left(\boldsymbol{v}_{G}-\boldsymbol{v}_{\mathrm{p} 1}\right) \cdot(\boldsymbol{u} \times \boldsymbol{B})+\boldsymbol{v}_{\mathrm{pl}} \cdot(\boldsymbol{u} \times \boldsymbol{B}) \\
& \quad=-E_{m}-\left[\boldsymbol{u}, \boldsymbol{v}_{\mathrm{pl}}, \boldsymbol{B}\right]
\end{aligned}
$$

where $v_{p l}$ is the inertial velocity of the ionospheric plasma at $G$ and the relation

$$
E_{m}=\left[\boldsymbol{u},\left(\boldsymbol{v}_{G}-v_{\mathrm{pl}}\right), \boldsymbol{B}\right]
$$

is used. This parameter, the induced electric field $E_{m}$, plays a relevant role in the electron-collection process. By definition, it will be positive for a passive tether and negative for an active one.

The integrals that are expressed in (27) can be rewritten as

$$
\begin{gathered}
\int_{-L(1-\mathfrak{G}(\gamma))}^{L G(\gamma)} I(x) \mathrm{d} x=I_{\mathrm{sc}} L_{*} \int_{0}^{\ell_{t}} i(\zeta) \mathrm{d} \zeta=I_{\mathrm{sc}} L_{*} U_{1} \\
\int_{-L(1-G(\gamma))}^{L \leftrightarrow(\gamma)} x I(x) \mathrm{d} x=I_{\mathrm{sc}} L_{*} \int_{0}^{\ell_{t}}\left(\ell_{t}(\xi(\gamma)-\zeta) i(\zeta) \mathrm{d} \zeta=I_{\mathrm{sc}} L_{*} \ell_{t}^{2} \hat{f}\right.
\end{gathered}
$$

In these expressions, the following nondimensional parameters are used:

$$
\begin{gathered}
U_{1}=\int_{0}^{\ell_{5}} i(\zeta) \mathrm{d} \zeta \\
U_{2}=\int_{0}^{\ell_{t}} \zeta i(\zeta) \mathrm{d} \zeta \\
\hat{f}=\frac{U_{1}}{\ell_{t}} \circlearrowleft(\gamma)-\frac{U_{2}}{\ell_{t}^{2}}
\end{gathered}
$$


Therefore, the power developed by the electrodynamic forces can be expressed in a compact relationship:

$$
\dot{W}_{f \boldsymbol{e}}=-E_{m} L_{*} I_{\mathrm{sc}} U_{1}-L_{*} I_{\mathrm{sc}} U_{1}\left[\boldsymbol{u}, \boldsymbol{v}_{\mathrm{pl}}, \boldsymbol{B}\right]-L_{*}^{2} I_{\mathrm{sc}} \ell_{t}^{2} \hat{f}[\boldsymbol{u}, \dot{\boldsymbol{u}}, \boldsymbol{B}]
$$

The right-hand side of Eq. (38) involves three terms. The first is the most important and summarizes the power developed by the Lorentz force, that is, by the current flowing along the cable. This term will be negative (positive) for passive (active) tethers due to the sign of $E_{m}$. The second term arises from the nonzero inertial velocity of the plasma. Again, the sign depends on the relative configuration of the vectors $\boldsymbol{u}, v_{p l}$, and $\boldsymbol{B}$. The last term relates to the attitude dynamics and the Lorentz torque; it vanishes for a zero Lorentz torque.

These three terms and their nondimensional forms turn out to be

$$
\begin{gathered}
\left.\dot{W}_{f e}\right|_{m}=-\left.E_{m} L_{*} I_{\mathrm{sc}} U_{1} \Rightarrow \dot{\tilde{W}}_{f e}\right|_{p}=\frac{\left.\dot{W}_{f e}\right|_{p}}{\left|E_{m}\right| \mathrm{LI}_{\mathrm{sc}}}=\mp \frac{U_{1}}{\ell_{t}} \\
\left.\dot{W}_{f e}\right|_{c}=-\left.L_{*} I_{\mathrm{sc}} U_{1}\left[\boldsymbol{u}, \boldsymbol{v}_{\mathrm{pl}}, \boldsymbol{B}\right] \Rightarrow \dot{\tilde{W}}_{f e}\right|_{c} \\
=\frac{\left.\dot{W}_{f e}\right|_{c}}{\left|E_{m}\right| \mathrm{LI}_{\mathrm{sc}}}=-\frac{U_{1}}{\ell_{t}} \frac{\left[\boldsymbol{u}, \boldsymbol{v}_{\mathrm{pl}}, \boldsymbol{B}\right]}{\left|E_{m}\right|}
\end{gathered}
$$

$$
\left.\dot{W}_{f e}\right|_{r}=-\left.L_{*}^{2} I_{\mathrm{sc}} \ell_{t}^{2} \hat{f}[\boldsymbol{u}, \dot{\boldsymbol{u}}, \boldsymbol{B}] \Rightarrow \dot{\tilde{W}}_{f e}\right|_{r}=\frac{\left.\dot{W}_{f e}\right|_{r}}{E_{m} \mathrm{LI}_{\mathrm{sc}}}=-\hat{f} L \frac{[\boldsymbol{u}, \dot{\boldsymbol{u}}, \boldsymbol{B}]}{\left|E_{m}\right|}
$$

where the parameter $\left|E_{m}\right| \mathrm{LI}_{\mathrm{sc}}$ has been introduced. The negative sign in Eq. (39) corresponds to passive tethers and the positive sign to active tethers.

The dimensionless expressions of the three terms allow us to establish the influence of the primary parameters. Note that the first (39) and second (40) terms are proportional to $U_{1} / \ell_{t}=\eta_{t}$, which is defined as the deorbiting efficiency [19] and depends only on the current collection. The third term (41) is proportional to $\hat{f}$, which is a parameter that appears in stability studies of relative motion and is proportional to the Lorentz torque with respect to the center of mass of the system. This parameter facilitates the definition of the concept of self-balanced EDT [20](SBET), because an SBET is an EDT with $\hat{f}=0$ for nominal environmental conditions.

From the preceding deduction and Eq. (27), it is straightforward to derive that $\left.\dot{W}_{f e}\right|_{p}+\left.\dot{W}_{f e}\right|_{c}=\boldsymbol{F}_{p} \cdot \boldsymbol{v}_{G}$, and $\left.\dot{W}_{f e}\right|_{r}=\boldsymbol{M}_{p} \cdot \omega$. Therefore, the first two terms (39) and (40) are responsible for the variation of the orbital energy of the center of mass $G$ of the system, whereas the third term (41) provides the variation of the rotational energy with respect to the center of mass.

Despite the convenience of these nondimensional expressions, they do not permit quantitative comparisons due to variations in $E_{m}$ (and therefore $I_{s}$ ) with the orbital and attitude conditions of the cable. It is better to use an alternative quantity as the characteristic magnitude, $E_{m}^{\text {ref }}$, that has a constant, fixed value. The mean value of $E_{m}$, as obtained in a hypothetical equatorial circular orbit with a radius that is equal to the equatorial terrestrial radius, was chosen to be $E_{m}^{\text {ref }}\left(E_{m}^{\text {ref }} \approx 260 \mathrm{~V} / \mathrm{km}\right)$. From a practical point of view, this is the maximum value that a passive tether can obtain in a circular orbit around the Earth. Consequently, the factor $E_{m}^{\text {ref }} L I_{\mathrm{sc}}^{\text {ref }}$ has a clear physical meaning: it is the maximum electrodynamic power that can be passively achieved with a certain cable following a circular Earth orbit. This parameter plays an important role in the design of the system, because it permits a better assessment of the actual performance of the tether. Moreover, this formulation allows the establishment of the specific power of the tether, which is the relationship between the maximum power and the mass of the cable:

$$
\dot{W}_{f e}^{\max }=E_{m}^{\mathrm{ref}} L I_{\mathrm{sc}}^{\mathrm{ref}}=\left(E_{m}^{\mathrm{ref}}\right)^{2} \sigma L A_{\mathrm{tc}}
$$

$$
M_{t}=\rho_{c} L A_{\mathrm{tc}}+\rho_{\mathrm{nc}} L A_{\mathrm{tnc}}
$$

where $\rho_{c}$ and $\rho_{\mathrm{nc}}$ are the densities of the conductive and nonconductive materials, respectively. Likewise, $A_{\mathrm{tc}}$ and $A_{\mathrm{tnc}}$ are the transversal areas of the conductive and nonconductive materials, respectively. When there is no insulation $\left(A_{\mathrm{tnc}}=0\right)$, the relation depends only on the chosen material:

$$
\frac{\dot{W}_{f e}^{\max }}{M_{t}}=\frac{\left(E_{m}^{\mathrm{ref}}\right)^{2} \sigma L A_{\mathrm{tc}}}{\rho_{c} L A_{\mathrm{tc}}}=\left(E_{m}^{\mathrm{ref}}\right)^{2} \frac{\sigma}{\rho}
$$

For example, for aluminum,

$$
\frac{\dot{W}_{f e}^{\max }}{M_{t}}=937.2 \frac{\mathrm{W}}{\mathrm{kg}}
$$

The inverse of this value, that is, the inverse specific power, would be about $1.1 \mathrm{~kg} / \mathrm{kW}$, which is consistent with previous estimations of this parameter. Using the factor $E_{m}^{\mathrm{ref}} \mathrm{LI}_{\mathrm{sc}}^{\mathrm{ref}}$ as the characteristic value, the terms contained in the expressions (39-41) for the electrodynamic power take the form

$$
\begin{gathered}
\frac{\left.\dot{W}_{f e}\right|_{p}}{E_{m}^{\mathrm{ref}} \mathrm{LI}_{\mathrm{sc}}^{\mathrm{ref}}}=-\left(\frac{E_{m}}{E_{m}^{\mathrm{ref}}}\right)^{2} \eta_{t} \\
\frac{\left.\dot{W}_{f e}\right|_{c}}{E_{m}^{\mathrm{ref}} \mathrm{LI}_{\mathrm{sc}}^{\mathrm{ref}}}=-\frac{E_{m}}{E_{m}^{\mathrm{ref}}} \frac{\left[\boldsymbol{u}, \boldsymbol{v}_{\mathrm{pl}}, \boldsymbol{B}\right]}{E_{m}^{\mathrm{ref}}} \eta_{t} \\
\frac{\left.\dot{W}_{f e}\right|_{r}}{E_{m}^{\mathrm{ref}} \mathbf{L} \mathbf{I}_{\mathrm{sc}}^{\mathrm{ref}}}=-\frac{E_{m}}{E_{m}^{\mathrm{ref}}} \frac{L[\boldsymbol{u}, \dot{\boldsymbol{u}}, \boldsymbol{B}]}{E_{m}^{\mathrm{ref}}} \hat{f}
\end{gathered}
$$

The parameters involved in these relations have the following dependencies:

$$
\begin{gathered}
\eta_{t}=\eta_{t}\left(\ell_{t}, \Omega, \tilde{V}_{\mathrm{cc}}\right) \\
\hat{f}=\hat{f}\left(\ell_{t}, \Omega, \tilde{V}_{\mathrm{cc}} ; \gamma\right) \\
\frac{E_{m}}{E_{m}^{\mathrm{ref}}}=\frac{E_{m}}{E_{m}^{\mathrm{ref}}}\left(a, e, i\{\text { or } b\}, \Omega_{\mathrm{an}}, \omega, v ; \phi, \theta\right) \\
\frac{\left[\boldsymbol{u}, \boldsymbol{v}_{\mathrm{pl}}, \boldsymbol{B}\right]}{E_{m}^{\mathrm{ref}}}=\frac{\left[\boldsymbol{u}, \boldsymbol{v}_{\mathrm{pl}}, \boldsymbol{B}\right]}{E_{m}^{\mathrm{ref}}}\left(a, e, i\{o r b\}, \Omega_{\mathrm{an}}, \omega, v ; \phi, \theta\right) \\
\frac{[\boldsymbol{u}, \dot{\boldsymbol{u}}, \boldsymbol{B}]}{E_{m}^{\mathrm{ref}}}=\frac{[\boldsymbol{u}, \dot{\boldsymbol{u}}, \boldsymbol{B}]}{E_{m}^{\mathrm{ref}}}\left(a, e, i\{o r b\}, \Omega_{\mathrm{an}}, \omega, v ; \phi, \theta, \dot{\phi}, \dot{\theta}\right)
\end{gathered}
$$

where $a, e, i\{o r b\}, \Omega_{\text {an }}, \omega$, and $v$ are the classical orbital elements of the center of mass and the libration angles of $\theta$ and $\phi$ that describe the attitude of the tether in the orbital frame. Consequently, and taking into account the tether dynamics, tether design and electrical variables influence the electrodynamic power through the two parameters $\eta_{t}$ and $\hat{f}$, which are related to variation in the orbital energy and the rotational energy, respectively. The actual values of the power are affected by terms that depend on orbital and attitude variables.

\section{Energy Bricks}

In the previous section, the effects of the electrodynamic forces on the tethered system were analyzed. In short, a passive tether reduces the mechanical energy of the system, whereas, conversely, an active tether increases the mechanical energy (even though the corotating plasma can play an important role and invert this behavior). There is 
also an impact on tether attitude dynamics that is proportional to the factor $\hat{f}$.

Nevertheless, there is another point of view to be considered in this analysis: the study of the different sinks and sources of energy that are present in the operation of the tether. These are called energy bricks because they build the power of the electrodynamic forces. This formulation elucidates the understanding of the tether as an energyexchange device.

The sinks and/or sources of electrodynamic power include ionospheric impedance, the cathodic contactor, ohmic losses, the interposed load, the power generator, and the corotational electric field. In the following, we briefly analyze each of these energy bricks.

\section{A. Ionospheric Impedance}

In the electron-collection process, the ionosphere presents an impedance, that is, a fraction of the power is spent in attracting electric charges from the surrounding plasma to the tether. The power can be computed as

$$
\begin{gathered}
\dot{W}_{\mathrm{II}}=-\int \Phi \mathrm{d} I=-E_{m} L I_{\mathrm{sc}} \frac{1}{\ell_{t}} \int \varphi \mathrm{d} i \\
\Rightarrow \dot{\tilde{W}}_{\mathrm{II}}=\frac{\dot{W}_{\mathrm{II}}}{\left|E_{m}\right| L I_{\mathrm{sc}}}=-\frac{1}{\ell_{t}} \int \varphi \mathrm{d} i
\end{gathered}
$$

The integral on the right-hand side of this expression is a line integral that is defined in the phase plane $(\varphi, i)$, and the path of integration is the curve $\varphi(i)$, which is the solution of the boundary-value problem of the tether-current profile. In nondimensional variables and for the passive regime, this can be written as

$$
\dot{\tilde{W}}_{\mathrm{II}}=-\frac{1}{\ell_{t}}\left[\left.\varphi \dot{i}\right|_{A} ^{C}-\int_{\varphi_{A}}^{\varphi_{C}} i(\varphi) \mathrm{d} \varphi\right]=\frac{\left|\varphi_{C}\right| i_{C}}{\ell_{t}}-\frac{1}{\ell_{t}} \int_{\varphi_{C}}^{\varphi_{A}} i(\varphi) \mathrm{d} \varphi
$$

For the active regime, and assuming that there is an insulated segment where the current collection vanishes, we have

$$
\dot{\tilde{W}}_{\mathrm{II}}=-\frac{1}{\ell_{t}}\left[\int_{\varphi_{C}}^{\varphi_{D}} i(\varphi) \mathrm{d} \varphi-\left.\varphi i\right|_{C} ^{D}\right]=\frac{\varphi_{D} \dot{i}_{D}}{\ell_{t}}-\frac{1}{\ell_{t}} \int_{\varphi_{C}}^{\varphi_{D}} i(\varphi) \mathrm{d} \varphi
$$

\section{B. Cathodic Contactor}

The power dissipated in the cathodic contactor is given by

$$
\dot{W}_{\mathrm{CC}}=-I_{C} V_{\mathrm{cc}}=-I_{\mathrm{sc}} E_{m} L i_{C} \tilde{V}_{\mathrm{cc}}
$$

In nondimensional variables, this will be

$$
\dot{\tilde{W}}_{\mathrm{CC}}=\mp i_{C} \tilde{V}_{\mathrm{cc}}
$$

where a positive value corresponds to the active regime.

\section{Ohmic Losses in the Tether}

The dissipated power in the conductive cable is given by

$$
\dot{W}_{\mathrm{OL}}=-\int_{0}^{R_{T}} I^{2} \mathrm{~d} R=-\int_{0}^{L} I^{2} \frac{1}{\sigma A_{t}} \mathrm{~d} h
$$

where $\mathrm{d} R=\mathrm{d} h /\left(\sigma A_{t}\right)$. In nondimensional terms,

$$
\dot{W}_{\mathrm{OL}}=-I_{\mathrm{sc}} E_{m} \sigma A_{t} \frac{1}{L_{\star}} \int_{0}^{\ell_{s}} i^{2}(\xi) \mathrm{d} \xi=-I_{\mathrm{sc}} E_{m} L \frac{1}{\ell_{t}} \int_{0}^{\ell_{t}} i^{2}(\xi) \mathrm{d} \xi
$$

Because for a passive tether $i=\mathrm{d} \varphi / \mathrm{d} \xi+1$, the last integral provides

$$
\begin{aligned}
& \dot{\tilde{W}}_{\mathrm{OL}}=-\frac{1}{l_{t}}\left[\int_{0}^{\ell_{\mathrm{s}}} i(\xi) \mathrm{d} \xi+\int_{\varphi_{A}}^{\varphi_{C}} i(\varphi) \mathrm{d} \varphi\right]=-\frac{U_{1}}{\ell_{t}} \\
& +\frac{1}{\ell_{t}} \int_{\varphi_{C}}^{\varphi_{A}} i(\varphi) \mathrm{d} \varphi
\end{aligned}
$$

In the active regime, in turn, the relation $i=\mathrm{d} \varphi / \mathrm{d} \xi-1$ holds for the bare segment and $i=i_{D}$ for the insulated stretch. The nondimensional expression of the ohmic losses along the cable is

$$
\begin{aligned}
\dot{\tilde{W}}_{\mathrm{OL}}= & -\frac{1}{l_{t}}\left[\int_{0}^{\ell_{D}} i(\xi) \mathrm{d} \xi-\int_{\varphi_{C}}^{\varphi_{D}} i(\varphi) \mathrm{d} \varphi-i_{D}^{2}\left(\ell_{t}-\ell_{D}\right)\right]= \\
& =-\frac{U_{1}}{\ell_{t}}+\frac{1}{\ell_{t}} \int_{\varphi_{C}}^{\varphi_{D}} i(\varphi) \mathrm{d} \varphi+\frac{\ell_{t}-\ell_{D}}{\ell_{t}} i_{D}\left(1+i_{D}\right)
\end{aligned}
$$

\section{Useful Power: Ohmic Losses in the Interposed Load}

The interposed load plays a double role. First, it can be used to model the useful power that is extracted from the tether, and, second, it can be used to control the current that is collected in the tether. In either case, the losses in this load should be assessed.

For passive tethers, the dissipated power in the interposed load is given by

$$
\begin{aligned}
\dot{W}_{\mathrm{IL}} & =-I_{C}^{2} Z_{T}=-I_{\mathrm{sc}}^{2} R_{T} i_{C}^{2} \Omega=-I_{\mathrm{sc}} E_{m} \sigma A_{t} \frac{L}{\sigma A_{t}} i_{C}^{2} \Omega \\
& =-I_{\mathrm{sc}} E_{m} L i_{C}^{2} \Omega
\end{aligned}
$$

By applying the circuit equation, in nondimensional variables, this relation takes the form

$$
\dot{\tilde{W}}_{\mathrm{IL}}=i_{C}\left(\tilde{V}_{\mathrm{cc}}-\frac{\left|\varphi_{C}\right|}{\ell_{t}}\right)
$$

This power can be used for onboard equipment or batteries and, for that reason, it should be considered useful power. The nondimensional variable $\dot{\tilde{W}}_{\mathrm{IL}}$ is a function of the nondimensional electrical parameters of $\Omega, \ell_{t}$, and $\tilde{V}_{\mathrm{cc}}$. Therefore, it is possible to represent the values of this nondimensional power, as can be observed in Fig. 3. Note that there is a maximum (close to 0.2 ), which appears for values of $\left(\ell_{t}, \Omega\right)$ in the neighborhood of $\Omega \approx 1$ and $\ell_{t} \approx 10$. This should be the design point when the primary objective is the recovery of part of the mechanical energy of the system. Notice that this "optimum" takes place in the "long-tether regime" $\left(\ell_{t}>4\right)$ introduced in [10]. In that study, the goal was to tune the tether for deorbiting a satellite as

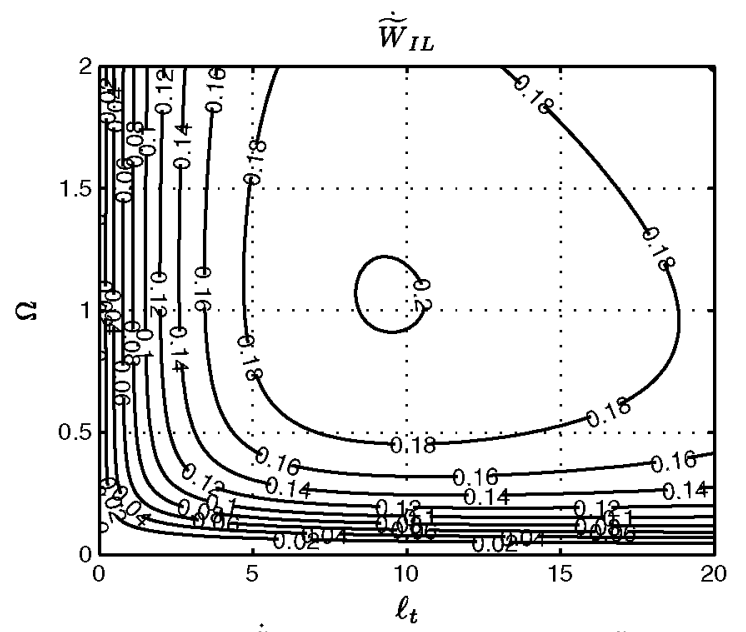

Fig. 3 Level curves of $\dot{\tilde{W}}_{\text {IL }}$ in the plane $\left(\ell_{i}, \Omega\right)$ for $\tilde{V}_{\mathrm{cc}}=0.1$. The qualitative behavior is similar for different values of $\tilde{V}_{\text {cc }}$. Note the maximum that is close to $\Omega \approx 1 ; \ell_{t} \approx 10$. 
rapidly as possible. Here, a different approach is adopted because a significant fraction of the power that is dissipated in the tether can be recovered as useful energy to be used onboard. Obviously, the deorbiting time will be longer in this case.

Figure 3 shows an interesting behavior: for small values of $\Omega$, the useful power is almost insensitive to changes in $\ell_{t}$ over a wide region of the long-tether regime $\left(\ell_{t}>4\right)$. Similarly, for small values of $\ell_{t}$, this power is almost insensitive to $\Omega$.

\section{E. Power Generator}

In the active regime, that is, to invert the natural direction of the tether current, an appropriate voltage must be applied. Therein, the required power supply can be computed as

$$
\dot{W}_{\mathrm{PG}}=-I_{A} \cdot \epsilon=-E_{m} I_{\mathrm{sc}} L i_{A} \tilde{\epsilon}
$$

or, in nondimensional variables, using the circuit equation,

$$
\dot{\tilde{W}}_{\mathrm{PG}}=\left(i_{D} \tilde{V}_{\mathrm{cc}}+i_{D}\left(1+i_{D}\right) \frac{\ell_{t}-\ell_{D}}{\ell_{t}}+\frac{\varphi_{D}}{\ell_{t}} i_{D}\right)
$$

\section{F. Corotational Electric Field}

There is a corotational electric field that is associated with the movement of the surrounding plasma relative to the inertial frame. Assuming that the plasma corotates with the Earth (see [21]), the value of this field can be calculated as

$$
\boldsymbol{E}=-\left(\boldsymbol{\omega}_{e} \times \boldsymbol{r}\right) \times \boldsymbol{B}
$$

where $\omega_{e}$ is the angular velocity of the Earth.

In some cases, this electric field is responsible for the dissipation of part of the energy of the system; in other cases, on the contrary, it can supply additional power. The power developed by such an electric field is given by

$$
\boldsymbol{F}=q \boldsymbol{E}=-q\left(\omega_{e} \times \boldsymbol{r}\right) \times \boldsymbol{B}
$$

$$
\dot{W}_{\mathrm{CF}}^{d s}=\boldsymbol{F} \cdot \boldsymbol{v}_{e}=-\boldsymbol{v}_{e} \cdot\left(q\left(\omega_{e} \times \boldsymbol{r}\right) \times \boldsymbol{B}\right)=I \boldsymbol{u} \cdot\left(\left(\omega_{e} \times \boldsymbol{r}\right) \times \boldsymbol{B}\right)
$$

where $q$ is the electronic charge, $v_{e}$ is the velocity of the electrons in the cable and the expression $-q v_{e}=I \boldsymbol{u}$ is used. Because the plasma moves with the angular velocity of the Earth, $v_{\mathrm{pl}}=\omega_{e} \times r$, the total power will therefore be

$$
\dot{W}_{\mathrm{CF}}=\int_{0}^{L} I(s) \mathrm{d} s \boldsymbol{u} \cdot\left(v_{\mathrm{pl}} \times \boldsymbol{B}\right)=I_{\mathrm{sc}} L_{*} U_{1}\left[\boldsymbol{u}, v_{\mathrm{pl}}, \boldsymbol{B}\right]
$$

or, in nondimensional terms,

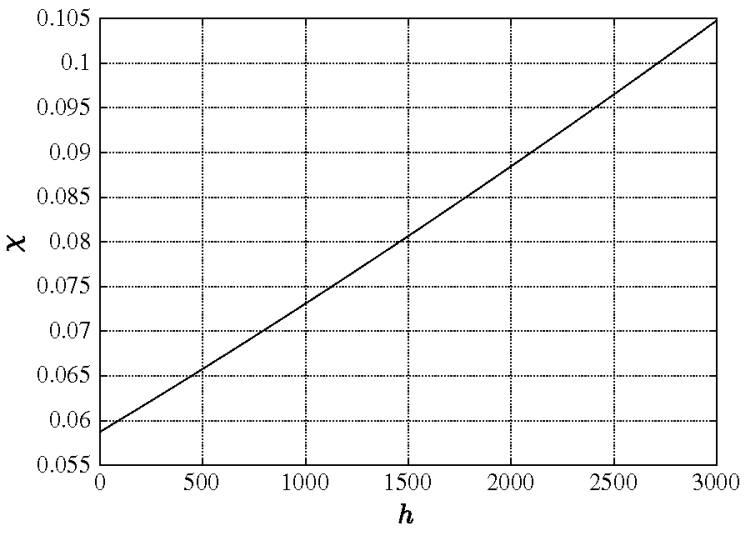

Fig. $4 \chi$ as a function of orbital altitude $h$ in $(\mathbf{k m})$ for a tether that is aligned with the local vertical, in a circular equatorial orbit.

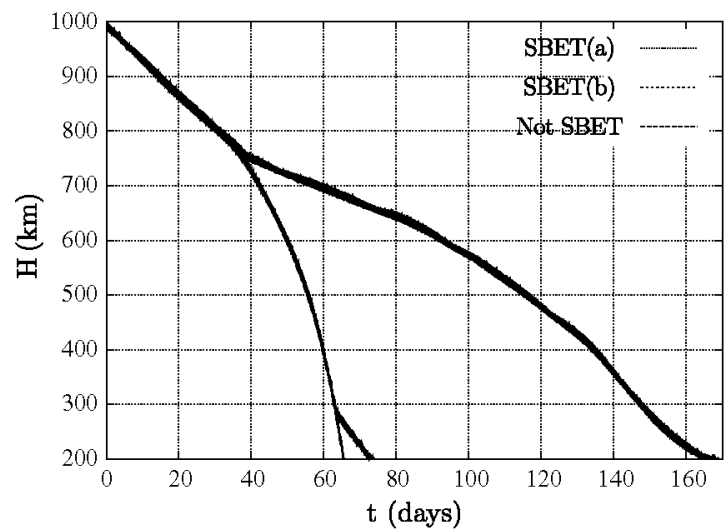

Fig. 5 Perigee altitude, $H$, vs time of descent.

$$
\dot{\tilde{W}}_{\mathrm{CF}}=\frac{U_{1}}{l_{t}} \cdot \chi \quad \text { where } \chi=\frac{\left[\boldsymbol{u}, \boldsymbol{v}_{\mathrm{pl}}, \boldsymbol{B}\right]}{\left|E_{m}\right|}
$$

The parameter $\chi$ is a function of the orbital elements and the attitude of the tether. Depending on its sign, the corotational plasma can subtract energy from or supply energy to the system. For Earth orbits, where the geostationary orbit is high above the limit of the ionosphere, it is not possible to obtain energy from the corotational electric field for a passive tether in a circular orbit, however, this is feasible in other orbits, for example, a Jovian orbit. For a further discussion of this issue, see [22].

The term $\dot{W}_{\mathrm{CF}}$ corresponds to $\left.\dot{\tilde{W}}_{f e}\right|_{c}$, which comes up naturally in the previous analysis. Figure 4 shows the behavior of the parameter $\chi$ in near-Earth circular orbits as a function of altitude variation. Several hypotheses were made to obtain this figure. First, it was assumed that the tether is in an equatorial circular orbit; therefore, $v_{G}$ and $v_{p l}$ are collinear. Moreover, it was assumed that the tether is aligned with the direction of the vector $\boldsymbol{v}_{G} \times \boldsymbol{B}$ (local vertical). As Fig. 4 shows, the value of $\chi$ increases quasi linearly with the altitude of the orbit and varies between 6 and $10 \%$.

\section{G. Power Balance}

All of the mechanisms discussed in this section must fulfill the requirement that the sum of the energy interchanges matches the computations made for the entire system.

For passive tethers, the sum of the dissipated power in every sink described in the previous paragraph results in

$$
\begin{aligned}
& \dot{\tilde{W}}_{\mathrm{II}}+\dot{\tilde{W}}_{\mathrm{CC}}+\dot{\tilde{W}}_{\mathrm{OL}}+\dot{\tilde{W}}_{\mathrm{IL}} \\
& \quad=\frac{\left|\varphi_{C}\right| \dot{i}_{C}}{\ell_{t}}-\frac{1}{\ell_{t}} \int_{\varphi_{C}}^{\varphi_{A}} i(\varphi) \mathrm{d} \varphi-i_{C} \tilde{V}_{\mathrm{cc}}-\frac{U_{1}}{\ell_{t}}+\frac{1}{\ell_{t}} \int_{\varphi_{C}}^{\varphi_{A}} i(\varphi) \mathrm{d} \varphi \\
& \quad+i_{C}\left(\frac{\tilde{V}_{\mathrm{cc}}-\left|\varphi_{C}\right|}{\ell_{t}}\right)=-\frac{U_{1}}{\ell_{t}}
\end{aligned}
$$

This is equal to the term $\left.\dot{\tilde{W}}_{f e}\right|_{p}$ of the power that is dissipated by the Lorentz force, as shown in Eq. (39); however, for active tethers, the equation is slightly different. In this case, the power supplied by the generator is partially dissipated but also provides mechanical energy. Using the previous terms, the power balance is expressed as

Table 1 Shared inputs of the numerical simulations

\begin{tabular}{ll}
\hline \hline Simulation characteristics & Input data \\
\hline Initial semimajor axis & $7378.1 \mathrm{~km}$ \\
Initial eccentricity & 0 \\
Initial inclination & $35^{\circ}$ \\
Initial date & $15 / 6 / 2000$ \\
Final semimajor axis & 6578.1 \\
\hline \hline
\end{tabular}


Table 2 Characteristics of the three different tether configurations

\begin{tabular}{lcccccccc}
\hline \hline Tether configuration & Mass $m_{1}$ & Mass $m_{2}$ & Mass $m_{c}$ & Total mass & Length & Diameter & Material & $V_{\mathrm{CC}}$ \\
\hline Self-balanced (a) & $647.8 \mathrm{~kg}$ & $392.2 \mathrm{~kg}$ & $0 \mathrm{~kg}$ & $1063 \mathrm{~kg}$ & $5 \mathrm{~km}$ & $1.5 \mathrm{~mm}$ & Aluminum & $10 \mathrm{~V}$ \\
Self-balanced (b) & $27.67 \mathrm{~kg}$ & $12.33 \mathrm{~kg}$ & $1000 \mathrm{~kg}$ & $1063 \mathrm{~kg}$ & $5 \mathrm{~km}$ & $1.5 \mathrm{~mm}$ & Aluminum & $10 \mathrm{~V}$ \\
Not balanced & $1000 \mathrm{~kg}$ & $40 \mathrm{~kg}$ & $0 \mathrm{~kg}$ & $1063 \mathrm{~kg}$ & $5 \mathrm{~km}$ & $1.5 \mathrm{~mm}$ & Aluminum & $10 \mathrm{~V}$ \\
\hline
\end{tabular}

$$
\begin{aligned}
& \dot{\tilde{W}}_{\mathrm{II}}+\dot{\tilde{W}}_{\mathrm{CC}}+\dot{\tilde{W}}_{\mathrm{OL}}+\left.\dot{\tilde{W}}_{f e}\right|_{p}=\frac{\varphi_{D} i_{D}}{\ell_{t}}+\frac{1}{\ell_{t}} \int_{\varphi_{C}}^{\varphi_{D}} i \mathrm{~d} \varphi+i_{A} \tilde{V}_{\mathrm{cc}} \\
& -\frac{U_{1}}{\ell_{t}}+\frac{1}{\ell_{t}} \int_{\varphi_{C}}^{\varphi_{D}} i \mathrm{~d} \varphi+\frac{\ell_{t}-\ell_{D}}{\ell_{t}} i_{D}\left(1+i_{D}\right)+\frac{U_{1}}{\ell_{t}}=\frac{\varphi_{D} \dot{i}_{D}}{\ell_{t}} \\
& \quad+i_{A} \tilde{V}_{\mathrm{cc}}+\frac{\ell_{t}-\ell_{D}}{\ell_{t}} i_{D}\left(1+i_{D}\right)=\dot{\tilde{W}}_{\mathrm{PG}}
\end{aligned}
$$

\section{Simulation Results}

In the context of deorbiting missions, the power developed by the electrodynamic forces and the mechanical energy sinks can be studied in the framework of the previous analysis. Therein, the aim was to determine the influence of tether mass configuration and electrical variables on the descent trajectory and energy balance.

Therefore, a series of numerical simulations were carried out that focused on a typical EDT in a midinclination orbit. These simulations were designed to assess the previous analysis and provide a first estimate of the possible capabilities of EDTs in a debris-mitigation mission. The characteristics of the simulations are compiled in the following.

The Runge-Kutta-Felberg 7/8 method was used to integrate the dynamic equations. The dynamic model of the tethered system is the dumbbell model, which retains the first librational mode of the tether

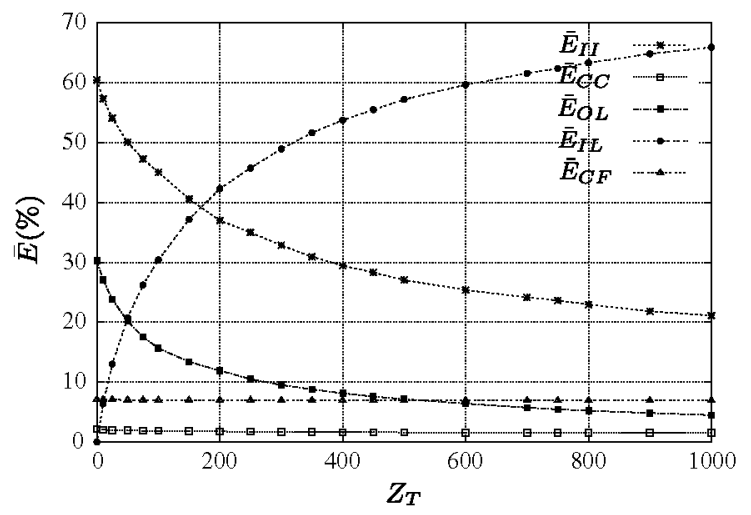

Fig. 6 The dissipated energy in each of the sinks described in the text vs the interposed load $Z_{T}$ (Ohms). The energy is expressed as a percentage of the total energy that is dissipated in the orbital transfer.

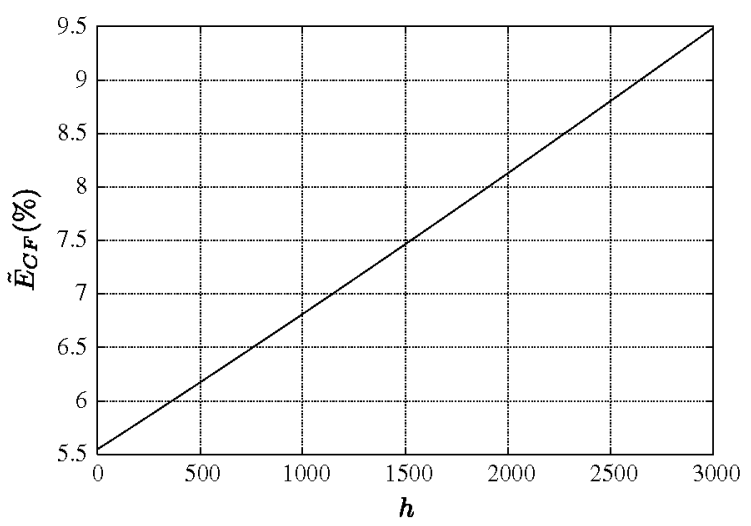

Fig. $7 \quad \tilde{E}_{\mathrm{CF}}$ as a function of orbital altitude $\boldsymbol{h}(\mathrm{km})$. and is thus well suited for the present purpose because the dynamic instability we are trying to avoid is related to this mode [23]. Nevertheless, it is worth noting that higher orders of the lateral motion as well as longitudinal motion are not modeled. Regarding the models of the environment, the International Reference Ionosphere model [24] is used for the ionosphere and the International Geomagnetic Reference Field for the magnetic field [25]. The atmospheric drag, in turn, is not considered in the simulations so as to avoid noise in the comparisons because they are focused exclusively on electrodynamic forces. The shared characteristics of all of these simulations are shown in Table 1. The dynamic initial conditions are fixed. Initially, the inclination is $35^{\circ}$, the orbit is circular, and the height is $1000 \mathrm{~km}$. The initial date was chosen to approximately coincide with a maximum of the solar cycle.

Regarding mass configuration, three options were considered; the tether characteristics are shown in Table 2. The total system mass, tether length, and tether diameter are the same in all of the tested cases. The differences in configuration are associated with the mass distribution. The self-balanced configuration (a) is an SBET with a high moment of inertia, because the entire mass is practically found at the ends. The self-balanced configuration (b) is also a SBET, although almost all of the mass is concentrated at the center of mass and, therefore, has a smaller moment of inertia. Finally, the third tether is unbalanced, because almost the entire mass is at one tether end. Regarding the electrical parameters, an interposed load was

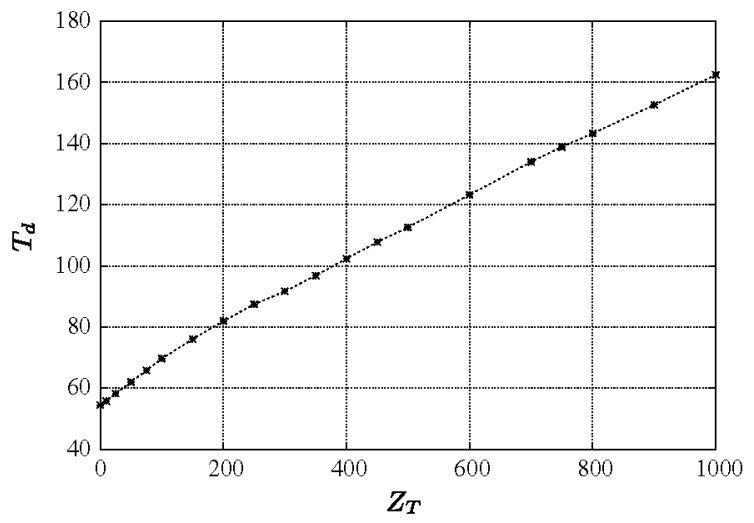

Fig. 8 Deorbiting time in days vs $Z_{T}$ (Ohms).

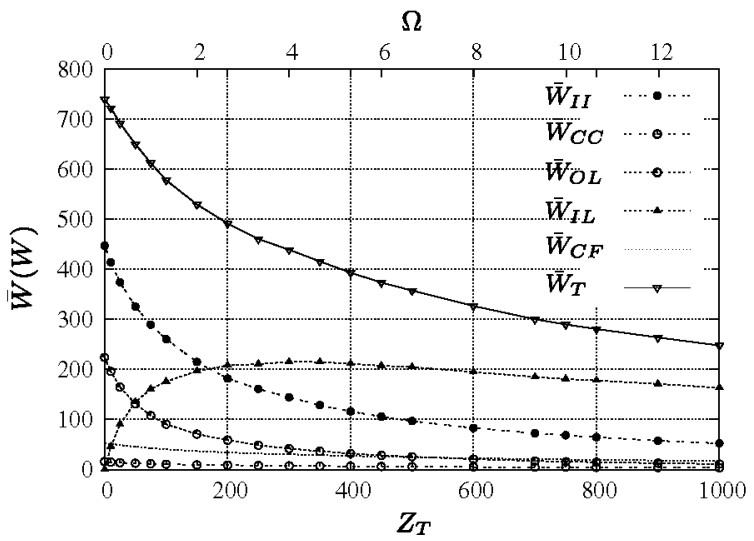

Fig. 9 Average power in watts vs $Z_{T}$ (Ohms). The upper axis corresponds to nondimensional values. 

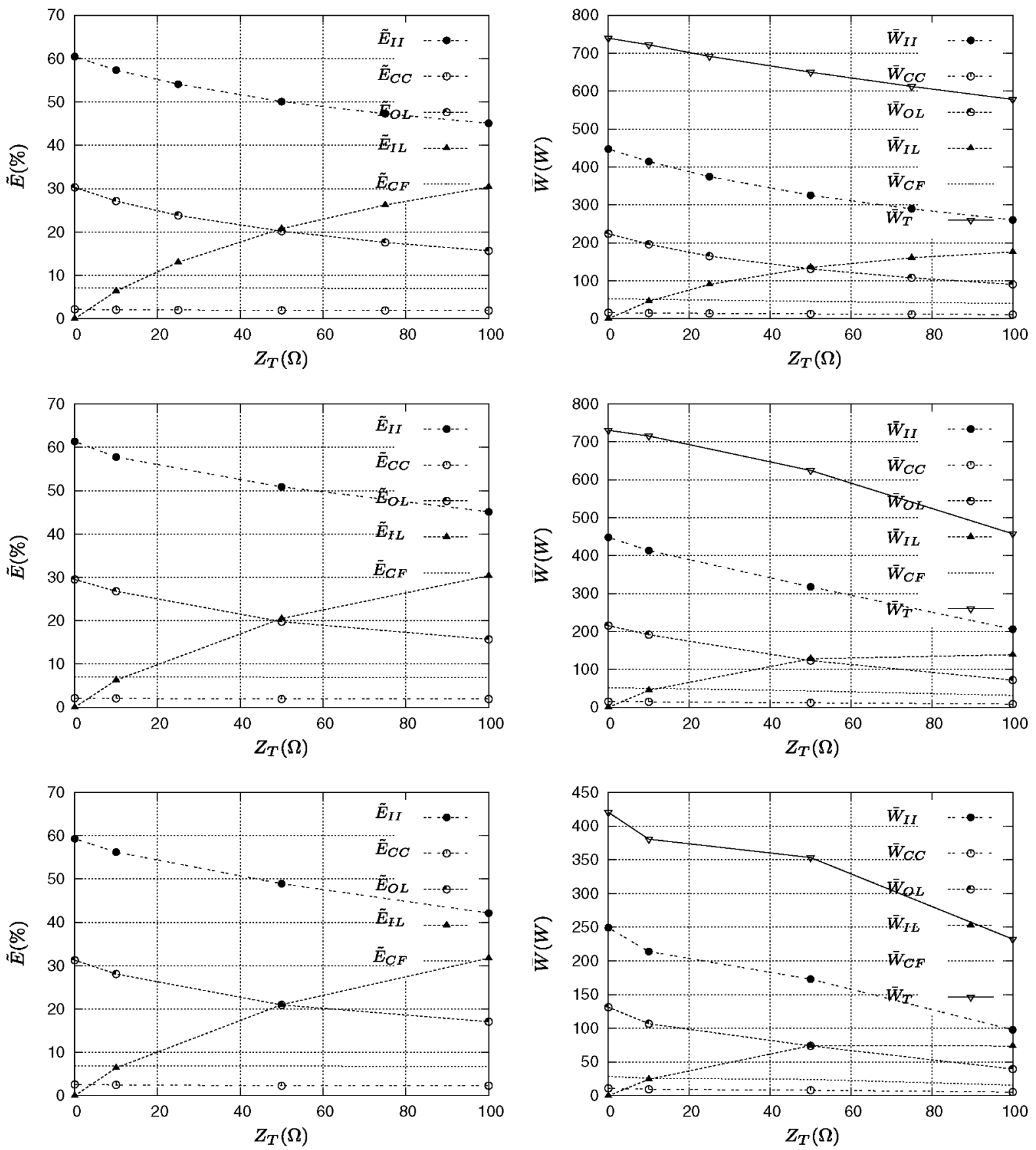

Fig. 10 The percentage of the total orbital energy dissipated by each sink and the average power for each configuration [SBET (a) in the first row, SBET (b) in the second, and non-SBET in the third].

used as a control variable and different values of this resistance were considered. The reference case to which the results of all of the simulations were compared is a self-balanced tether with the same mass configuration as that described in (a) and an interposed load of $100 \Omega$. In Fig. 5, the change in the perigee altitude during the descent trajectory for the three configurations is shown. A detailed exposition and analysis of the simulation campaign can be found in [26]. Here, some of the results are reproduced, wherein the interposed load was varied from $0 \Omega$ to $1000 \Omega$ for the same SBET configuration.

Let us consider the total energy that is dissipated during the orbital transfer:

$$
\Delta E_{M}=\int_{t_{i}}^{t_{f}}\left(\left.\dot{W}_{e f}\right|_{p}+\left.\dot{W}_{e f}\right|_{c}\right) \mathrm{d} t
$$

from an initial orbit at a $1000 \mathrm{~km}$ height to a final orbit at a $200 \mathrm{~km}$ height.
Figure 6 depicts the ratios of $\tilde{E}_{\alpha}=100 \cdot E_{\alpha} / \Delta E_{M}, \alpha=\mathrm{II}$ (ionospheric impedance), cathodic contactor (CC), ohmic looses (OL), interposed load (IL), and corotational field (CF), as a function of the interposed load $Z_{T}$. On the ordinate axis and for each of the previously considered mechanisms, we represent the percentage of the total energy lost in the descent, that is, the dissipated energy. It is clear that the useful energy, $\tilde{E}_{\mathrm{IL}}$, increases with $Z_{T}$; however, the other contributions of $\tilde{E}_{\alpha}, \alpha=\mathrm{II}, \mathrm{CC}$, and $\mathrm{OL}$ decrease, whereas $\tilde{E}_{\mathrm{CF}}$ remains approximately constant because $\dot{\tilde{W}}_{\mathrm{CF}}$ does not depend on the electrical parameters. In fact, it is possible to compute the variation in $\tilde{E}_{\mathrm{CF}}$ as a function of orbital altitude by taking into account the assumptions made in the previous section:

$$
\tilde{E}_{\mathrm{CF}}=\frac{E_{\mathrm{CF}}}{\Delta E_{M}}=\frac{\int_{t_{i}}^{t_{f}}\left(E_{m} \mathrm{LI}_{\mathrm{sc}} \dot{\tilde{W}}_{\mathrm{CF}}\right) \mathrm{d} t}{\int_{t_{f}}^{t_{f}}\left(\left.\dot{W}_{f e}\right|_{p}+\left.\dot{W}_{f e}\right|_{c}\right) \mathrm{d} t}
$$


By considering the mean values of the variables as a rough approximation of the integrals, it is possible to derive the value of $\tilde{E}_{\mathrm{CF}}$ as a function of the mean value of the parameter $\chi$ in the descent trajectory. Because $\chi$ is a function of $h, \tilde{E}_{\mathrm{CF}}$ is also a function of $h$ (see Fig. 7):

$$
\tilde{E}_{\mathrm{CF}} \approx \frac{\langle\chi\rangle}{1+\langle\chi\rangle}
$$

The distribution of mechanical energy in each energy brick is not the most suitable parameter for measuring the performance of the EDT. Note that the time of descent is another significant feature that has not been considered. The ability of the EDT to recover a significant part of the power that is dissipated in the orbital descent is not free of cost. If we tune the tether parameters so as to increase the useful energy that can be obtained in the descent, the time of descent increases. Note that the useful energy, $\tilde{E}_{\text {II }}$, increases with $Z_{T}$, as shown in Fig. 6. Equally, the time of descent, $T_{d}$, increases with $Z_{T}$, as shown in Fig. 8, which summarizes the results of our simulations. To include this important parameter, we consider the average power that is dissipated by each sink in the entire orbital transfer because, this averaged value involves the time of descent and is closely related to operational issues of the tether. The average power is defined as $\bar{W}=E_{\alpha} / T_{d}, \alpha=\mathrm{II}, \mathrm{CC}, \mathrm{OL}, \mathrm{IL}$, and CF. The behavior of these averaged powers is shown in Fig. 9. The average useful power, $\bar{W}_{\mathrm{IL}}$, presents a maximum that lies between approximately 350 and $500 \mathrm{Ohms}$. It should be noted that in the neighborhood of its maximum, the curve $\bar{W}_{\mathrm{IL}}=\bar{W}_{\mathbb{I}}\left(Z_{T}\right)$ is very flat. This fact facilitates working near the maximum value of the useful power for a large interval of $Z_{T}$. Because the interposed load also plays a role in other aspects of tether operation, the existence of this large interval of $Z_{T}$ that leads to a near-optimum useful power makes the required tradeoff easier. In our simulations, the total dissipated power varied between 700 and $250 \mathrm{~W}$. If we consider the mass of the cable, $23 \mathrm{~kg}$, the specific power provided is then between 30.4 and $10.9 \mathrm{~W} / \mathrm{kg}$, and represents 3.24 and $1.15 \%$, respectively, of the maximum that can be obtained with an aluminum tether [see Eq. (45)]. Changes in the mass configuration lead to relevant variations in the descent trajectory. When the tether is not balanced, it starts to rotate at some point. Because of the nonzero value of $\hat{f}$, a fraction of the work of the electrodynamic forces is spent on changing the rotational state of the tether. Obviously, from a practical point of view, this behavior is unacceptable; however, we also present the results of those simulations for comparison. Figure 10 shows the mean energy as a function of the interposed load for each configuration [SBET (a), SBET (b), and non-SBET]. These results show that $\tilde{E}_{i}$ does not depend on the mass configuration of the system. Note that for these simulations the initial and final orbits are fixed; therefore, the total mechanical energy loss is the same (the terminal condition has been imposed in terms of the semimajor axis). The time history of each of the energy sinks depends on environmental conditions, through the nondimensional variables $\Omega, \tilde{V}_{\mathrm{cc}}, \ell_{t}$ and the parameter $\chi$. Therefore,

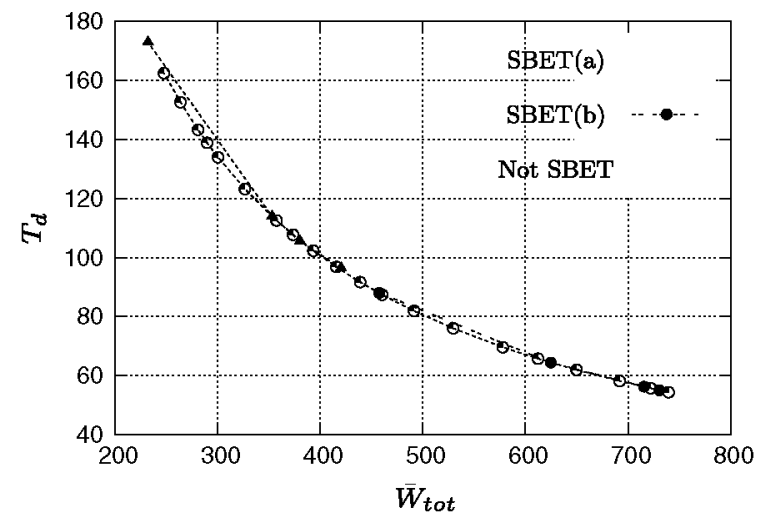

Fig. 11 The time of descent in days vs dissipated power $\bar{W}_{\text {tot }}$ for different configurations. when descent trajectories are similar, the respective energy percentages of each sink are also similar, which is the case for the SBET (a) and (b) configurations, however, the simulations show the same results for the non-SBET case despite it having a significantly different descent trajectory (see Fig. 5). This suggests that environmental conditions do not have a relevant effect on the energy distribution.

Nonetheless, the global average power was deeply affected by changes in the mass configuration, as can be seen in the right column of Fig. 10 (note the different scales of the ordinate axis). This substantial decrease in power is due to an increase in the descent time $T_{d}$, as the value of the energy remained the same. This time of descent becomes thus a meaningful parameter: it measures two aspects of performance, the descent time itself and power, in a single parameter. Indeed, for each energy sink, the average power fulfills this relationship:

$$
\bar{W}_{i}=\frac{E_{i}}{T_{d}} \Leftrightarrow \bar{W}_{i} T_{d}=E_{i}
$$

As a consequence, the influence of the mass configuration on the global average power $\bar{W}_{\text {tot }}$ and $T_{d}$ should be balanced because the total energy lost, $\Delta E$, is constant. Thus, the previous expression leads to a hyperbola.

Figure 11 describes this interesting property. Here, the time of descent in days is plotted against the global average power, $\bar{W}_{\text {tot }}$, that is dissipated during the descent process.

The three mass configurations that were considered in these simulations are included in the figure. Note that all of the points in the simulations can be included in a hypothetical hyperbola. Nevertheless, only a part of the entire hyperbola is accessible for any given mass configuration, wherein SBET (a) provides the greatest flexibility. Moreover, this figure describes the requirements of the tradeoff between the time of descent and useful power, as the useful power of the fastest descent is zero.

\section{Conclusions}

This energy analysis highlights the role of EDTs as energy converters. The study of the different sources and sinks of electrical and mechanical energy provides insight into the operation of the tether. In particular, power generation, thrust generation and librational motion can be understood in a global context. Moreover, these aspects of passive and active tether performance can be investigated and their dependence on the design and environmental parameters can be elucidated.

Regarding the numerical simulations presented herein, three aspects of tether performance must be considered in missions that involve the descent of space debris: 1) the velocity of the descent, measured by the orbital decay or the descent time, $T_{d} ; 2$ ) the dynamic stability of the tethered system during the descent process; and 3) in those cases that imply the retrieval of energy, the amount of energy that can be recovered.

In the section that was devoted to the power of the electrodynamic force, it was shown that the three facets are linked and can be analyzed from an energy viewpoint.

The first variable, descent time (or the global dissipated power because they are related) is the most important measure of the deorbiting efficiency of the transfer-orbit maneuver. Descent time depends on the electrical parameters, environmental conditions and attitude dynamics. In the literature, it has been stated that the best strategy in a deorbiting mission is maximization of the deorbiting parameter $\eta_{t}$. This means that there is no interposed load in which to maximize the current along the cable. Taking into account the obtained simulation results, the last statement is true when the attitude dynamics are not considered. In fact, when the librational motion of the tether is taken into account, the results show that having good attitude control could be more efficient than working with a high deorbiting parameter, although this control implies sacrificing some efficiency, that is, a lower value of $\eta_{t}$. Consequently, the 
relevance of providing control strategies is stressed, and the importance of having good control of the attitude dynamics is clear.

Finally, regarding the third feature of tether performance, power generation, there exists a value of the interposed load $Z_{T}$ that maximizes the useful power that can be obtained. Note that the power curve is very flat in the neighborhood of its maximum, which permits operation over a broad interval of interposed loads. This is convenient because the interposed load also influences the dynamics and the descent time, and it is necessary to make tradeoffs among all of these aspects.

\section{Acknowledgments}

This work was conducted in the framework of the research project entitled Propagation of Orbits, Advanced Orbital Dynamics and Use of Space Tethers (ESP2007-64068) supported by the Dirección General de Investigación of the Spanish Ministry of Science and Technology.

\section{References}

[1] Drell, S. D., Foley, H. M., and Ruderman, M. A., "Drag and Propulsion of Large Satellites in the Ionosphere: An Alfvén Propulsion Engine in Space," Physical Review Letters, Vol. 14, No. 6, Feb. 1965, pp. 171175 . doi:10.1103/PhysRevLett.14.171

[2] Alfven, H., "Spacecraft Propulsion: New Methods," Science, Vol. 176, No. 4031, 1972, pp. 167-168. doi: $10.1126 /$ science.176.4031.167

[3] Martínez-Sánchez, M., and Hastings, D. E., "A Systems Study of a $100 \mathrm{~kW}$ Electrodynamic Tether," Joumal of the Astronautical Sciences, Vol. 35, No. 1, 1987, pp. 75-96.

[4] Levin, E. M., Dynamic Analysis of Space Tether Missions, American Astronautical Society, San Diego, CA, 2007.

[5] Cartmell, M. P., and McKenzie, D. J., "A Review of Space Tether Research," Progress in Aerospace Sciences, Vol. 44, No. 1, 2008, pp. 1-21. doi:10.1016/j.paerosci.2007.08.002

[6] Sanmartin, J. R., and Lorenzini, E. C., and Martinez-Sanchez, M., "A Review of Electrodynamic Tethers for Space Applications," AIAA Paper 2008-4595, 44th AIAA/ASME/SAE/ASEE Joint Propulsion Conference and Exhibit, July 2008.

[7] Stone, N. H., "Why Not Space Tethers? 2nd Spacecraft Propulsion Joint Subcommittee," NASA TR 20070031863, Marshall Space Flight Center, 2007.

[8] Iess, L., Bruno, C., Ulivieri, C., Ponzi, U., Parisse, M., Laneve, G., Vannaroni, G., Dobrowolny, M., De Venuto, F., Bertotti, B., and Anselmo, L., "Satellite Deorbiting by Means of Electrodynamic Tethers Part 1: General Concepts and Requirements," Acta Astronautica, Vol. 50, April 2002, pp. 399-406. doi:10.1016/S0094-5765(01)00180-1

[9] Iess, L., Bruno, C., Ulivieri, C., and Vannoroni, G., "Satellite DeOrbiting by Means of Electrodynamic Tethers Part 2: System Configuration and Performance," Acta Astronautica, Vol. 50, April 2002, pp. 407-416. doi:10.1016/S0094-5765(01)00181-3

[10] Ahedo, E., and Sanmartín, J. R., "Analysis of Bare-Tether Systems for Deorbiting Low-Earth-Orbit Satellites," Journal of Spacecraft and Rockets, Vol. 39, March 2002, pp. 198-205. doi: $10.2514 / 2.3820$
[11] Lorenzini, E. C., Estes, R. D., Cosmo, M. L., Sanmartin, J., Pelaez, J., and Ruiz, M., "The Propulsive Small Expendable Deployer System (ProSEDS)," Smithsonian Astrophysical Observatory Special Report, 2003, pp. 1-201.

[12] Forward, R. L., Hoyt, R. P., and Uphoff, C. W., "Terminator Tether Number 153: A Spacecraft Deorbit Device," Journal of Spacecraft and Rockets, Vol. 37, March 2000, pp. 187-196. doi: $10.2514 / 2.3565$

[13] Williams, P., "Optimal Orbital Maneuvers Using Electrodynamic Tethers," Advances in the Astronautical Sciences, Vol. 120, No. 2, 2005, pp. 1671-1690.

[14] Peláez, J., and Sanjurjo, M., "Generator Regime of Self Balanced Electrodynamic Tethers," Journal of Spacecraft and Rockets, Vol. 43, No. 6, Nov.-Dec. 2006, pp. 1359-1369. doi: $10.2514 / 1.20471$

[15] Sanmartín, J. R., Martínez-Sánchez, M., and Ahedo, E., "Bare Wire Anodes for Electrodynamic Tethers," Journal of Propulsion and Power, Vol. 9, No. 3, May-June 1993, pp. 353-360. doi: $10.2514 / 3.23629$

[16] Sanmartín, J. R., and, Estes, R. D., "The Orbital-Motion-Limited Regime of Cylindrical Langmuir Probes," Physics of Plasmas, Vol. 6, No. 1, Jan. 1999, pp. 395-405. doi:10.1063/1.873293

[17] Sanmartín, J. R., and Estes, R. D., "Magnetic Self-Field Effects on Current Collection by an Ionospheric Bare Tether," Journal of Geophysical Research [Space Phys.], Vol. 107, No. A11, Nov. 2002, p. 1335. doi:10.1029/2002JA009344

[18] Sanmartin, J. R., Charro, M., Pelaez, J., Tinao, I., Elaskar, S., Hilgers, A., and Martinez-Sanchez, M., "Floating Bare Tether as Upper Atmosphere Probe," Journal of Geophysical Research [Space Phys.], Vol. 111, Nov. 2006, pp. A11310. doi:10.1029/2006JA011624

[19] Ahedo, E., and Sanmartín, J. R., "Analysis of Electrodynamic Tethers as Deorbiting Systems," 36th Joint Propulsion Conference, AIAA Paper 2000-3263, Hunstville, AL, 16-19 July 2000.

[20] Peláez. J., "Self Balanced Electrodynamic Tethers," AIAA Paper 20045309, 2004 AAS/AIAA Astrodynamics Specialist Conference and Exhibit, Providence, RI, 2004.

[21] Sanmartin, J. R., "Macroscopic Motion and Gravitation in Thermodynamics," European Journal of Physics, Vol. 16, Jan. 1995, pp. $8-13$. doi:10.1088/0143-0807/16/1/002

[22] Sanmartín, J. R., and, Lorenzini, E. C., "Exploration of the Outer Planets Using Tethers for Power and Propulsion," Joumal of Propulsion and Power, Vol. 21, No. 3, May-June 2005, pp. 573-576. doi:10.2514/1.10772

[23] Pelaez, J., Lorenzini, E. C., López-Rebollal, O., and Ruiz, M., "A New Kind of Dynamic Instability in Electrodynamic Tethers," Journal of the Astronautical Sciences, Vol. 48, No. 4, 2000, pp. 449-476.

[24] Bilitza, D., Reinisch, B., and Lastovicka, J., "Progress in ObservationBased Ionospheric Modeling," Space Weather, Vol. 6, No. 2, p. S02002. doi:10.1029/2007SW000359

[25] http://www.ngdc.noaa.gov/IAGA/vmod/home.html [retrieved Sept. 2009].

[26] Sanjurjo-Rivo, M., "Self-Balanced Bare Electrodynamic Tethers. Space Debris Mitigation and Other Applications," Ph.D., Thesis, Universidad Politécnica de Madrid, Madrid, 2009. 